\title{
Repeat-pass SAR interferometry for land cover classification: a methodology using Sentinel-1 Short-Time-Series
}

\author{
Francescopaolo Sica, Andrea Pulella, Matteo Nannini, \\ Muriel Pinheiro, and Paola Rizzoli \\ Microwaves and Radar Institute, German Aerospace Center (DLR), Münchener Straße 20, \\ 82234 Weßling. \\ Corresponding author: Francescopaolo Sica, Email: Francescopaolo.Sica@dlr.de
}

\begin{abstract}
In this paper we explore the potential of repeat-pass interferometric SAR (InSAR) for land cover classification purposes. We introduce a novel approach for the generation of large-scale thematic maps, based on the use of multi-temporal data, acquired over short observation intervals (short-time-series). A larger interferometric coherence loss is expected with the increasing time difference between two interferometric acquisitions. This phenomenon is normally indicated as temporal decorrelation whose amount differs depending on the type of imaged target on ground. The basic idea is therefore to accurately model the evolution in time of the temporal decorrelation and use the estimated parameters, together with backscatter, as input features for the Random Forest machine learning classification algorithm. The work has been carried out on the case study of land cover mapping over central Europe, considering Sentinel-1 C-band interferometric stacks, acquired over a time span of about one month. Three different land cover classes have been considered: artificial surfaces as e.g. urban areas, forests, and non-forested areas as the ensemble of low vegetation, bare soil, and agricultural areas. The results show a level of agreement above $91 \%$, when compared to the CORINE land cover map product of 2012, which has been used as external reference for both training and testing of the classification algorithm.
\end{abstract}

Keywords: land cover classification, SAR, interferometric coherence, Sentinel-1,

Preprint submitted to Remote Sensing of Environment

June 24, 2019 
temporal decorrelation.

\section{1. Introduction}

$2 \quad$ The objective of land cover mapping is the classification of the (bio)physical

3 cover of the Earth's surface and is applied in many scientific and social/economic

4 spheres, such as land use monitoring, environmental planning, and resource

5 assessment. This task can be performed either by field measurements or by the

6 analysis of remotely sensed data. The first approach is very accurate but does not

7 allow for the generation of large-scale maps and is clearly confined to areas which

s can be easily accessed. Because of such limitations, and for its cost-efficiency,

9 the development of automatized algorithms for land cover classification using

10 remote sensing systems has become of paramount importance. Currently, various

11 large-scale and global products have been generated using space-borne optical

12 sensors, among which the GlobCover Map [1], derived from MERIS data, or

the MODIS Collection 5 global land cover [2. Moreover, temporal series of land cover maps can be utilized for detecting changes on ground and represent a helpful tool for monitoring dynamic changes occurring on the Earth surface, such as natural hazards and deforestation.

In particular, forests play a key role in the Earth's ecosystem. They help reducing the concentration of carbon dioxide in the atmosphere and controlling climate changes. In this framework, an effective monitoring of forests is of crucial importance, in order to detect possible degradation, caused by either natural events or human activities, such as selective logging or illegal deforestation. Nowadays, optical and laser sensors are widely used for mapping forests extent and changes [3], [4, [5].

Given the extended cloud coverage which can hide large areas from optical sensors during most of the year, radar spaceborne sensors, with their capabilities to acquire data independently on weather and daylight conditions, represent a necessary tool for providing a constant monitoring at a global scale. For this purpose, detected SAR backscatter is widely exploited for forest mapping and 

44 wide-swath (IW) mode at C band [14]. cross-polarization channel HV 9 .

land cover characterization [6], 7], 8]. The analysis of backscatter signature has led to the development of successful techniques and to the release of operational products, such as the global forest/non-forest map from L-band ALOS PALSAR data, which was generated by properly thresholding backscatter levels in the

The first experiments based on the use of the interferometric coherence for land cover classification relied on the use of ERS-1/2 data [10, [11, [12], [13]. More recently, the large availability of repeat-pass data with very precise orbit definition has allowed for a reliable use of SAR interferometry (InSAR) for many different applications, such as deformation and natural hazards monitoring or topography reconstruction. In this framework, the Sentinel-1 mission opened new avenues for land cover classification using time-series data. It comprises two satellites (Sentinel-1a and Sentinel-1b), which allow for a short revisit-time (12 or 6 days if one single or both satellites are considered, respectively) and it typically acquires large swaths of about $260 \mathrm{~km}$ in range using the interferometric

In this paper we aim to explore the potential of interferometric repeat-pass SAR for land cover classification purposes. We present a novel algorithm which exploits the synergies between backscatter and interferometric information to derive a reliable classification map of the observed scene.

Differently from traditional methods that exploit long time-series (with an observation interval which varies from several months up to years) and classify the target on the base of its backscatter temporal dynamic [12, [15, we shorten the temporal series to a set of data up to six acquisitions that, for a 6 -days revisit time, translates into one month acquisition interval. In the present work, we refer to such data as short-time-series, in order to highlight the reduced observation interval. Requiring a lower amount of data, the proposed approach abbreviates the usual idle-time, i.e. the time interval that goes from the retrieval of the first acquisition to the generation of the end product (the thematic map). This facet enhances the systematical mapping at regular intervals of the given target area, allowing for land cover monitoring and keeping track of abrupt cover change 
events such as deforestation phenomena or the establishment of new settlements. In the present paper, we show that the interferometric information is a valuable resource for the classification and that it can recoup the performance degradation due to the reduced stack size. Specifically, we combine a mathematical modeling of the temporal decorrelation contribution with the Random Forest machine learning algorithm and we show how the use of the multi-temporal interferometric coherence can improve the accuracy of the classification with respect to the case when the sole amplitude is utilized. For this purpose, we select a test case over central Europe, where the presence of both Sentinel-1a and -1b acquisitions allows for a 6-days revisit time analysis.

The paper is organized as follows: in section 2, we summarize a series of background concepts while in section 3 we describe the proposed methodology and the utilized data sets. In section 4 , we present and discuss the empirical results and finally, in section 5 , conclusions and outlook are drawn.

\section{Background}

SAR Interferometry employs at least two SAR acquisitions to retrieve information about the imaged scene, by exploiting a given acquisition diversity that depends on the nature of the phenomenon that has to be observed (geometry, time, frequency, etc.). While carrying the useful information, the dissimilarity between the two observations causes a degradation of the interferometric signal: intuitively, two SAR images acquired at two different time instants have likely a lower degree of similarity with respect to simultaneous acquisitions.

The interferometric coherence describes the degree of correlation between two SAR acquisitions and, for this reason, it represents the key parameter to assess the quality of an interferogram. It is defined as the amplitude of the complex correlation between the two images, named Single Look Complex (SLC). We indicate with $(x, y)$ the master and slave SLCs, respectively, then the 
interferometric coherence $\rho$ has the following expression:

$$
\rho=\frac{\left|E[x] E\left[y^{*}\right]\right|}{\sqrt{E\left[|x|^{2}\right] E\left[|y|^{2}\right]}},
$$

88 where $E[\cdot]$ is the mathematical expectation, $*$ the complex conjugate operator, 89 and $|\cdot|$ indicates the absolute value. The interferometric signal can be degraded 9o by various decorrelation sources. As shown in [16] and [17, the coherence can 91 be described as the product of single contributions as follows:

$$
\rho=\rho_{\text {SNR }} \rho_{\text {quant }} \rho_{\text {amb }} \rho_{\text {az }} \rho_{\text {rg }} \rho_{\text {vol }} \rho_{\text {temp }},
$$

92

where the different terms on the right-hand side identify the correlation factors due to limited SNR $\left(\rho_{\mathrm{SNR}}\right)$, quantization noise $\left(\rho_{\text {quant }}\right)$, ambiguities $\left(\rho_{\mathrm{amb}}\right)$, relative shift of the Doppler spectra $\left(\rho_{\mathrm{az}}\right)$, baseline decorrelation $\left(\rho_{\mathrm{rg}}\right)$, volume decorrelation $\left(\rho_{\mathrm{vol}}\right)$, and temporal decorrelation $\left(\rho_{\text {temp }}\right)$.

It is worth noting that the volume correlation factor $\rho_{\mathrm{vol}}$, which represents the amount of decorrelation occurring because of multiple reflections within a volume, has already been used for land classification purposes. This kind of decorrelation typically occurs in presence of vegetation and snow-covered areas, where the radar wave penetrates within the canopy and the snow pack, respectively. In case of single-pass interferometry, the coherence is not affected by temporal decorrelation, being $\rho_{\text {temp }}=1$. It is therefore possible to isolate $\rho_{\text {vol }}$ from all other contributions as:

$$
\rho_{\mathrm{vol}}=\frac{\rho}{\rho_{\mathrm{SNR}} \rho_{\text {quant }} \rho_{\mathrm{amb}} \rho_{\mathrm{az}} \rho_{\mathrm{rg}} \rho_{\mathrm{temp}}}
$$

and to use it as input feature for the classification. In this framework, examples are given by the global TanDEM-X Forest/Non-Forest Map [18] [19], or the classification of Greenland snow facies in [20].

In this paper, we focus on repeat-pass interferometry, where the interferometric pair is acquired at two different time instants, being therefore affected by temporal decorrelation. Hence, we aim to classify the observed target on ground on the basis of the evolution in time of its temporal correlation factor $\rho_{\text {temp }}$. 
Up to date, several works have been proposed in the literature to model the temporal decay of the interferometric coherence. Developed in the context of the estimation of the target temporal decorrelation in application to differential interferometry, the model in 21] describes the temporal evolution of the coherence for bare soil or lightly vegetated areas as:

$$
\rho(t)=\rho_{0} e^{-\frac{t}{\tau}}
$$

where $\rho_{0}$ is defined as the short-term coherence and takes into account all the decorrelation phenomena except from the temporal one. $\tau$ is the temporal decorrelation constant and indicates how fast the exponential decreases. This model has been further extended in [22] with the introduction of the long-term coherence term $\rho_{\mathrm{LT}}$, in order to consider the fact that a scatterer may not completely decorrelate, even after a long time:

$$
\rho(t)=\left(\rho_{0}-\rho_{\mathrm{LT}}\right) e^{-\frac{t}{\tau}}+\rho_{\mathrm{LT}}
$$

On the other hand, based on previous works on target decorrelation in along-track interferometry (ATI) applications [23], 24], a slightly different model appears in [17] and describes the temporal correlation factor $\rho_{\text {temp }}$ only as:

$$
\rho_{\text {temp }}(t)=e^{-\left(\frac{t}{\tau}\right)^{2}}
$$

As in equation 4, this model also describes the temporal decorrelation evolution over time as a decreasing exponential, but differs from the previous ones for the squared term at the exponent. For the present work, we combine the concept of the long-term coherence with the last model in equation 6, as presented in the next section.

\section{Methods and materials}

In this section, we present the developed methods. In section 3.1 we introduce and discuss the proposed model for the temporal correlation factor. In section 3.2 


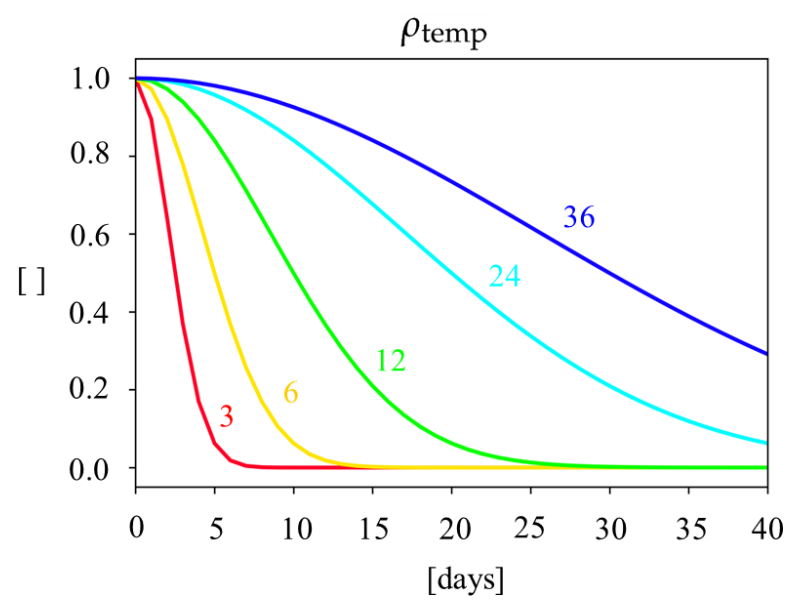

Figure 1: Exponential model of $\rho_{\text {temp }}$ as in equation 7, derived for $\rho_{\mathrm{LT}}=0$ and different values of $\tau$ (from 3 to 36 days).

144 shows the behavior of such a model for different values of $\tau$ and assuming

$145 \rho_{\mathrm{LT}}=0$.

$$
\rho_{\text {temp }}(t)=\left(1-\rho_{\mathrm{LT}}\right) e^{-\left(\frac{t}{\tau}\right)^{2}}+\rho_{\mathrm{LT}},
$$

with $\tau$ the target decorrelation factor and $\rho_{\mathrm{LT}}$ the long term coherence. As in 22, we added the latter term to the model in order to take into account that some targets may not completely decorrelate even after a long time. Figure 
As it can be observed from equation $7 \rho_{\text {temp }}$ equals 1 for $t=0$ and tends to $\rho_{\mathrm{LT}}$ for $t \rightarrow \infty$, while its decay velocity is regulated by the target decorrelation constant: a lower $\tau$ means a faster decay and viceversa. After a time interval $\tau$ the exponential function decreases from a value of 1 to $1 / e$ (where $e$ is the Neper constant). The sampling of the temporal correlation factor model is $t=n T$, where $T$ represents the satellite revisit time and $n \in[0, \infty[$.

The choice of this model is based on experimental observations, which aim to compare the fitting performance for the different models, presented in equation 4. 5. and 6, with respect to the proposed one. The result of this comparison is presented later on in section 4.1.1

\subsection{Sentinel-1 processing chain}

In the following, we describe the processing chain of Sentinel-1 (S-1) stacks, from the focused data to the retrieval of the interferometric parameters. We consider a stack of $M$ focused S-1 Interferometric Wide-Swath (IW) acquisitions, coregistered with respect to a common master geometry. The latter is chosen as the one closest to the central acquisition date of the entire stack, as usually done for differential interferometry applications. The coregistration of each SLC stack is performed as indicated in [25]. After a preliminary geometrical coregistration, the enhanced spectral diversity (ESD) technique is applied to the overlapping areas between subsequent bursts. This procedure allows for the achievement of a coregistration accuracy in the order of centimeters, i.e. a fraction of few thousands of the pixel size, and consequently for the absence of phase jumps between subsequent bursts. The effectiveness of this coregistration algorithm has been shown in [26], in application to differential interferometry and tomography with Sentinel-1 data.

We propose a processing strategy that allows for the combined use of backscatter and interferometric parameters. The block diagram in figure 2 shows the implemented processing chain. After the coregistration of the entire stack, the main branch is splitted into two sub-processing chains: the $S L C$ processing, for the estimation of the multi-temporal backscatter $\gamma^{0}$, and the InSAR processing, 


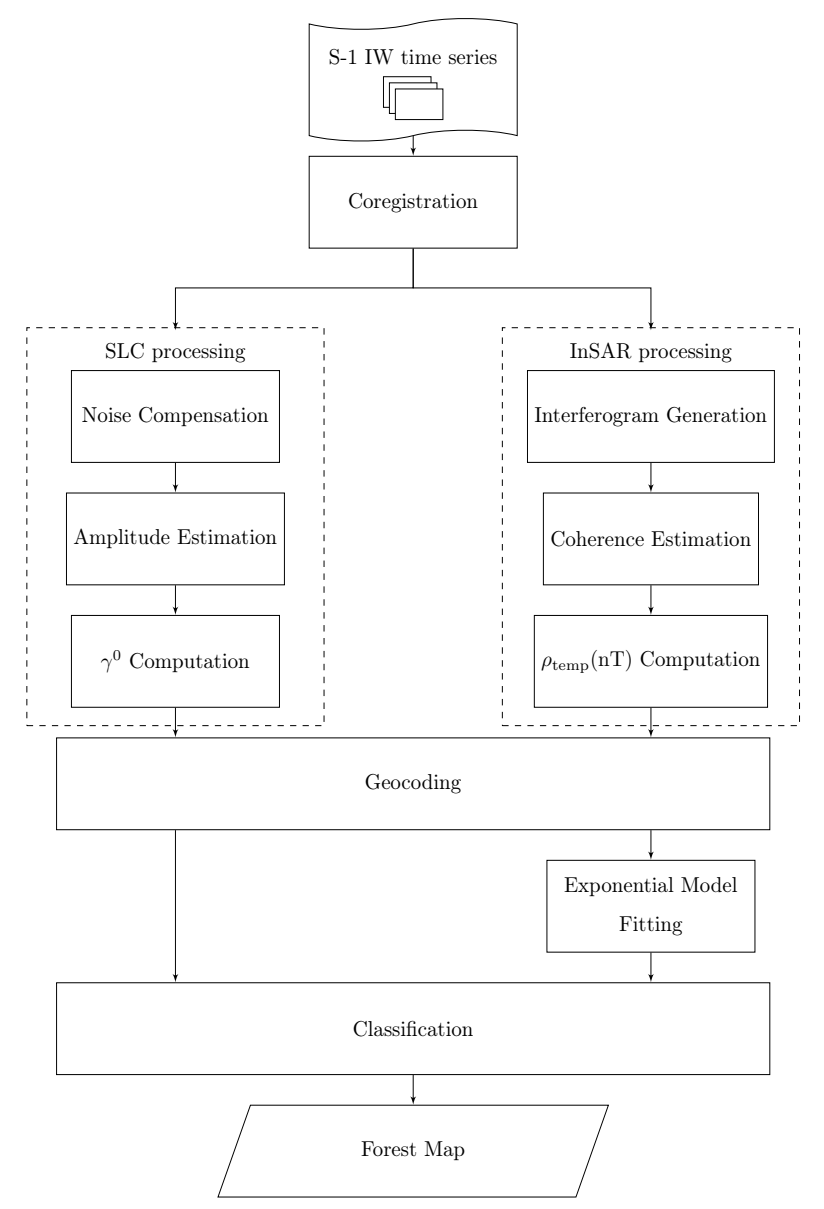

Figure 2: Sentinel-1 processing chain.

176 for the estimation of the temporal correlation factor. In this case, the retrieved

${ }_{177} \rho_{\text {temp }}$ is then projected over a $100 \mathrm{~m} \times 100 \mathrm{~m}$ geocoded grid, which matches 178 with the resolution of the external reference map, introduced later in section 1793.5 .2 Finally, the exponential fitting is performed along the time dimension.

\subsubsection{SLC processing}

In order to retrieve information on the land cover from the detected backscatter, the intrinsic reflectivity of the target should be measured independently of the time $(t)$. Indeed, land classification applications require an almost exclusive dependence of backscatter on the physical properties of the observed target [27], 
[28]. For this purpose we apply the radiometric correction to the S-1 Digital Number (the effectively annotated SLC value) and compute the gamma nought coefficient $\gamma^{0}$. In support of this approach, it is worth mentioning that the gamma nought has been already successfully exploited for land cover purposes, such as forest mapping at L band [9] or snow facies classification at X band [20]. In order to retrieve the multi-temporal $\gamma^{0}$, we first remove the system noise floor (noise equivalent sigma nought) by using the designated Look-Up-Table (LUT) provided within S-1 data. We estimate the amplitude image $A_{m}$ of the $m^{\text {th }}$ SLC ( $m \in[0, M[$ ) by assuming local spatial stationarity and applying a $7 \times 27$ pixels moving average filter:

$$
\hat{A}_{m}[p]=\sqrt{\sum_{i \in \Omega(p)} A_{m}^{2}[i]}
$$

where $p$ is the current estimated pixel, and $\Omega(p)$ a $7 \times 27$ boxcar window around $p$. Note that the window size is chosen accordingly to the azimuth and ground range resolution of Sentinel-1 interferometric wide-swath (IW) data: $14 \mathrm{~m}$ and $3.7 \mathrm{~m}$, respectively [29]. A window size of $7 \times 27$ pixels assures a product resolution of circa $100 \mathrm{~m} \times 100 \mathrm{~m}$, which matches with the external reference map (introduced later on in section 3.5.2.

For the sake of simplicity and when not strictly necessary, we use in the text from now on only one index to indicate bi-dimensional image coordinates. Hence the $\gamma^{0}$ is computed by means of the local incidence angle $\theta_{\text {inc }}$ and the calibration factor $K$ as:

$$
\hat{\gamma}_{m}^{0}=K \hat{A}_{m} \tan \left(\theta_{\mathrm{inc}}\right),
$$

where $\hat{\gamma}_{m}^{0}$ represents the derived $\gamma^{0}$ for the $m^{\text {th }}$ image within the stack. In order to get a unique value of backscatter, $\hat{\gamma}^{0}$, representative for the whole stack, we finally average along the third dimension of the stack (time) all the computed 

$\hat{\gamma}_{m}^{0}$ as:

$$
\hat{\gamma}^{0}=\sum_{m=1}^{M} \hat{\gamma}_{m}^{0}
$$

\subsubsection{InSAR processing}

Given the stack of $M$ SLCs, we generate all the interferograms within a given temporal baseline of $N \cdot T$ days, with $\max (N)=M-1$. All the available interferograms have hence a temporal baseline given by $\Delta t=n \cdot T$ with $n \in[1, N]$. Before the coherence estimation, we apply the common-band filter in azimuth and range [30], in order to avoid decorrelation due to spectral shift and baseline.

We assume now the local stationarity of the interferometric signal and estimate the coherence with a $7 \times 27$ pixels moving average filter as:

$$
\hat{\rho}[p]=\frac{\left|\sum_{i \in \Omega(p)} x[i] y[i]^{*}\right|}{\sqrt{\sum_{i \in \Omega(p)}|x[i]|^{2} \sum_{i \in \Omega(p)}|y[i]|^{2}}} .
$$

By means of the relation between coherence, number of looks, and bias [31, we further compensate for the bias within the coherence estimation.

The temporal correlation factor $\rho_{\text {temp }}$ can be finally isolated from the interferometric coherence by inverting equation 2 . In our specific case, the different contributions are quantified in the following way:

- $\rho_{\mathrm{SNR}}$ : is estimated through the expression [17]:

$$
\hat{\rho}_{\mathrm{SNR}}=\frac{1}{\sqrt{\left(1+\mathrm{SNR}_{1}^{-1}\right)\left(1+\mathrm{SNR}_{2}^{-1}\right)}},
$$

where $\mathrm{SNR}_{1}$ and $\mathrm{SNR}_{2}$ are the signal-to-noise ratios of the master and slave images, respectively, calculated by considering the derived gamma nought from the different images and the corresponding annotated noise profiles 32 .

- $\rho_{\text {quant }}$ : the used FDBAQ quantization scheme adapts the number of quantization bits to the local backscatter level in order to minimize the signal-toquantization noise ratio [33. Given the high performance of the algorithm, we assume that this contribution is close to 1 and therefore negligible. 
- $\rho_{\mathrm{amb}}$ : the corresponding coherence loss can be approximated by [17]:

$$
\rho_{\mathrm{amb}}=\frac{1}{(1+\mathrm{AASR})} \frac{1}{(1+\mathrm{RASR})},
$$

where AASR and RASR are the azimuth and range ambiguity-to-signal ratios, respectively. In the case of $\mathrm{S}-1$, the azimuth and range distributed target-to-ambiguity ratios are provided [34] (az-DTAR and rg - DTAR, respectively). In particular, for IW mode, the worst case shows az - DTAR $=-25.29 \mathrm{~dB}(\mathrm{IW} 1 \mathrm{subswath})$ and $\mathrm{rg}-\mathrm{DTAR}=-26.10$ dB (IW3 subswath). This values, when applied to equation 13 , lead to $\rho_{\mathrm{amb}}=0.99$, which can therefore be neglected.

- $\rho_{\mathrm{az}}$ and $\rho_{\mathrm{rg}}$ : this kind of decorrelations are compensated by applying a common-bandwidth azimuth and range filter during the generation of the interferogram, leading therefore to $\rho_{\mathrm{az}}=1$ and $\rho_{\mathrm{rg}}=1$.

- $\rho_{\mathrm{vol}}$ : given S-1 small orbital tube of only $50 \mathrm{~m}$ radius [14, the volume correlation factor can be neglected $\left(\rho_{\mathrm{vol}}=1\right)$ [24. This assumption is also sustained by experimental observations from the analysis of X-band bistatic TanDEM-X data in [35], where it was observed that, for such small baselines, no significant decorrelation is detected.

Therefore, given all considerations above, we finally derive the temporal correlation factor $\hat{\rho}_{\text {temp }}$ from the estimated coherence $\hat{\rho}$ as:

$$
\hat{\rho}_{\text {temp }}=\frac{\hat{\rho}}{\hat{\rho}_{\mathrm{SNR}}} .
$$

\subsection{Exponential model fitting}

At this stage, the complete set of temporal correlation factors for the entire stack is computed and we map them to a $100 \mathrm{~m} \times 100 \mathrm{~m}$ georeferenced grid. Figure 3 shows in matrix form all the available correlation values (visually represented as green cells) for the generic pixel $p$ on ground. We exploit all the available interferometric pairs by setting $N=N_{\mathrm{MAX}}=5$ and hence allow the 


\section{[days]}

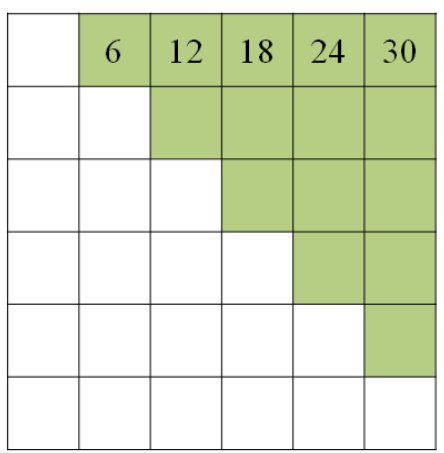

Figure 3: Available correlation values for a point on ground (green cells) for $M=6$ and $N=5$.

number of generated interferograms to reach a maximal temporal baseline of $N \cdot T=30$ days.

For every pixel on ground $p$ we define now the tensor of all computed temporal correlation values $\hat{\rho}_{\text {temp }}[n, i, j]$, where $n \in[1, N]$ spans the temporal axis, $i \in[1, N-n]$ spans all the available values for a given temporal baseline $n \cdot T$, and $j \in \Omega(p)$ spans the spatial axis in a square neighborhood $\Omega(p)$ of size $L$ around the current estimated pixel.

Before applying the model fitting we identify those pixels which, because of strong decorrelation phenomena, loose the monotonic decreasing trend along time and show a particularly noisy behavior. In order to overcome this limitation, for these pixels we consider a larger spatial neighborhood $(L=5)$, while for all the others $L=1$.

Subsequently, the model fitting is performed with a least square approach by numerically solving the following functional:

$\left(\hat{\tau}, \hat{\rho}_{\mathrm{LT}}\right)=\underset{\tau, \rho_{\mathrm{LT}}}{\arg \min }\left\{\sum_{n=1}^{N} \sum_{i=1}^{N-n} \sum_{j \in \Omega(p)}\left(\left(1-\rho_{\mathrm{LT}}\right) e^{-\left(\frac{n T}{\tau}\right)^{2}}+\rho_{\mathrm{LT}}-\hat{\rho}_{\mathrm{temp}}[n, i, j]\right)^{2}\right\}$,

where $\hat{\tau}$ and $\hat{\rho}_{\mathrm{LT}}$ are the estimated target decorrelation constant and long term 
coherence, respectively.

\subsection{Classification approach}

In this section, we provide a description of the used approach to face the classification task. We exploit the Random Forest (RF) classifier, a very powerful machine learning algorithm that provides high classification accuracy while requiring a very low number of input parameters [36]. The RF algorithm is non-parametric and, indeed, no assumption has to be made on the form of the mapping function, allowing for a high flexibility of the algorithm when generalized to unseen data. This last property is very important to our task, since remotely sensed data may slightly differ for a given class depending on the environmental conditions at the acquisition time.

In applications related to land cover classification, the use of the RF algorithm is relatively recent and has been proven to be a very effective tool for optical, multi/hyper-spectral, and SAR data [37, [38, [39, [40, [41].

Moreover, we aim to quantify the impact that multi-temporal interferometric parameters have on the classification performance. For this purpose, we apply the $\mathrm{RF}$ algorithm with different input features:

- case 1: $\hat{\gamma}^{0}$, and $\theta_{\text {inc }}$,

- case 2: $\hat{\tau}, \hat{\rho}_{\mathrm{LT}}$, and $\theta_{\mathrm{inc}}$,

- case 3: $\hat{\gamma}^{0}, \hat{\tau}, \hat{\rho}_{\mathrm{LT}}$, and $\theta_{\mathrm{inc}}$.

The local incidence angle $\theta_{\text {inc }}$ is a very important feature since it carries information on the SAR acquisition geometry. Indeed, it merges the topography information and the satellite position at the moment of the acquisition. By adding this parameter, the typical backscatter dependency from the side-looking nature of SAR sensors, can be correctly taken into account by the RF algorithm.

In all cases, given the relatively small number of input features, we let the $\mathrm{RF}$ algorithm to use them all for each of the created trees. We further use the Gini index [42] to minimize the probability of misclassification and set the 
number of estimators (number of trees in the forest) as well as the minimum number of samples in a leaf node (leaf size) to 50. These last parameters have been experimentally chosen after a preliminary performance analysis, which is presented later on in section 4.1 .2 .

In this work, we classify $P=3$ different land cover classes, as detailed in section 3.5 A more diversified classification with a larger number of classes can also be achieved by exploiting the same proposed framework. This will be objective of future investigations.

\subsubsection{Performance evaluation}

In order to assess the classification performance, we compare the derived classification map over the selected test site with an external reference map.

Here, one can derive the confusion matrix $C$, which has the following form:

$$
C=\left[\begin{array}{ccc}
c_{1,1} & c_{1, j} & c_{1, P} \\
c_{i, 1} & c_{i, j} & c_{i, P} \\
c_{P, 1} & c_{P, j} & c_{P, P}
\end{array}\right] .
$$

$C$ is a $P \times P$ table layout ( $P$ is the total number of classes), where each row spanned by the index $i$ ) represents the instances in an estimated class, while each column (spanned by the index $j$ ) represents the instances in the reference class. In particular, the total number of pixels for the $j^{\text {th }}$ class $N_{j}$ is given by:

$$
N_{j}=\sum_{i=1}^{P} c_{i, j}
$$

and the overall accuracy $A$ is then defined as:

$$
A=\frac{\sum_{j=1}^{P} c_{j, j}}{\sum_{j=1}^{P} N_{j}} .
$$

\subsection{Materials}

For the present work, we considered a large test site located in central Europe and, in particular, over Germany. The area, depicted in figure 4 , extends by about $700 \mathrm{~km} \times 500 \mathrm{~km}$. The used data sets are described in the following. 
Table 1: Description of the considered Sentinel-1 multi-temporal data set over central Europe (Germany). For each stack, the following parameters are displayed: relative orbit number, geographical region and region abbreviation (Abbrev.), acquisition dates of the single images, and corner coordinates in latitude (lat) and longitude (lon). For each stack, the symbol* indicates the master image.

\begin{tabular}{|c|c|c|c|c|c|c|c|}
\hline & Stack 1 & Stack 2 & Stack 3 & Stack 4 & Stack 5 & Stack 6 & Stack 7 \\
\hline orbit & 139 & 139 & 139 & 168 & 168 & 168 & 168 \\
\hline region & Baden-Württemberg & Rheinland-Palatinate & Nord Rhein-Westphalen & Bayern & Thüringen & Sachsen & Mecklenburg-Vorp. \\
\hline Abbrev. & BW & $\mathrm{RP}$ & NW & BY & $\mathrm{TH}$ & SN & MV \\
\hline Image & \multicolumn{7}{|c|}{ Acquisition dates } \\
\hline 1 & 2018.08 .01 & 2018.08 .01 & 2018.08 .01 & 2018.07 .28 & 2018.07 .28 & 2018.07 .28 & 2018.07 .28 \\
\hline 2 & 2018.08 .07 & 2018.08 .07 & 2018.08 .07 & 2018.08 .03 & 2018.08 .03 & 2018.08 .03 & 2018.08 .03 \\
\hline 3 & $2018.08 .13^{*}$ & $2018.08 .13^{*}$ & $2018.08 .13^{*}$ & 2018.08 .09 & 2018.08.09 & 2018.08.09 & 2018.08.09 \\
\hline 4 & 2018.08 .19 & 2018.08 .19 & 2018.08 .19 & $2018.08 .15^{*}$ & $2018.08 .15^{*}$ & $2018.08 .15^{*}$ & $2018.08 .15^{*}$ \\
\hline 5 & 2018.08 .25 & 2018.08 .25 & 2018.08 .25 & 2018.08 .21 & 2018.08 .21 & 2018.08 .21 & 2018.08 .21 \\
\hline \multirow[t]{2}{*}{6} & 2018.08 .31 & 2018.08 .31 & 2018.08 .31 & 2018.08 .27 & 2018.08 .27 & 2018.08 .27 & 2018.08 .27 \\
\hline & \multicolumn{7}{|c|}{ Corner Coordinates [deg] } \\
\hline lat $\min$ & 47.9676283 & 49.4508966 & 50.9358976 & 47.9499978 & 49.4358306 & 50.9199976 & 52.4016654 \\
\hline lat $\max$ & 49.4748923 & 50.9608575 & 52.4458574 & 49.9545851 & 51.4413178 & 52.9263836 & 54.4098489 \\
\hline lon min & 5.238333 & 5.5870266 & 5.9489214 & 9.3441662 & 9.6941661 & 10.0491661 & 10.4188564 \\
\hline lon $\max$ & 8.9128063 & 9.3759577 & 9.8550366 & 13.1737098 & 13.6414315 & 14.1314738 & 14.6397917 \\
\hline
\end{tabular}

\subsubsection{Sentinel-1 Data Set}

We considered seven stacks of Sentinel-1 IW scenes (VV polarization channel), each of those comprising 6 acquisitions characterized by a revisit time of 6 days and covering an overall time span of one Month (August 2018). The acquisition orbits, dates, and geographical coordinates of the utilized stacks are summarized in table 1 Each input IW image, composed by three sub-swaths, covers an area in range of $260 \mathrm{~km}$ at a resolution of $14 \mathrm{~m} \times 3.7 \mathrm{~m}$ in the azimuth and ground range dimensions, respectively.

\subsubsection{The CORINE land cover reference map}

As external reference land cover map, we used the CORINE Land Cover Map from 2012 [43. It consists of an inventory of 44 land cover classes generated by visual inspection from IRS P6 LISS III and RapidEye dual date satellite data. The product has a pixel spacing of $100 \mathrm{~m} \times 100 \mathrm{~m}$ and a thematic accuracy higher than $85 \%$. The delivered classes are defined using a three-layer hierarchical nomenclature and are summarized in table 2 .

For the purposes of the present investigation, we grouped such classes into four 
Table 2: Higher-level class grouping from CORINE (ART: artificial surfaces, FOR: forests, NFR: non-forested areas, INV: water bodies and invalid or no data).

\begin{tabular}{|c|c|c|c|}
\hline & CORINE Labels & & \\
\hline Label 1 & Label 2 & Label 3 & Higher-level class \\
\hline Artificial surfaces & Urban fabric & Continuous urban fabric & \\
\hline Artificial surfaces & Urban fabric & Discontinuous urban fabric & \\
\hline Artificial surfaces & Industrial, commercial and transport units & Industrial or commercial units & \\
\hline Artificial surfaces & Industrial, commercial and transport units & Road and rail networks and associated land & \\
\hline Artificial surfaces & Industrial, commercial and transport units & Port areas & \\
\hline Artificial surfaces & Industrial, commercial and transport units & Airports & ART \\
\hline Artificial surfaces & Mine, dump and construction sites & Mineral extraction sites & \\
\hline Artificial surfaces & Mine, dump and construction sites & Dump sites & \\
\hline Artificial surfaces & Mine, dump and construction sites & Construction sites & \\
\hline Artificial surfaces & Artificial, non-agricultural vegetated areas & Green urban areas & \\
\hline Artificial surfaces & Artificial, non-agricultural vegetated areas & Sport and leisure facilities & \\
\hline Forest and semi natural areas & Forests & Agro-forestry areas & \\
\hline Forest and semi natural areas & Forests & Agro-forestry areas & FOR \\
\hline Forest and semi natural areas & Forests & Coniferous forest & \\
\hline Agricultural areas & Arable land & Non-irrigated arable land & \\
\hline Agricultural areas & Arable land & Permanently irrigated land & \\
\hline Agricultural areas & Arable land & Rice fields & \\
\hline Agricultural areas & Permanent crops & Vineyards & \\
\hline Agricultural areas & Permanent crops & Fruit trees and berry plantations & \\
\hline Agricultural areas & Permanent crops & Olive groves & \\
\hline Agricultural areas & Pastures & Pastures & \\
\hline Agricultural areas & Heterogeneous agricultural areas & Annual crops associated with permanent crops & \\
\hline Agricultural areas & Heterogeneous agricultural areas & Complex cultivation patterns & \\
\hline Agricultural areas & Heterogeneous agricultural areas & Land principally occupied by agriculture... & \\
\hline Agricultural areas & Heterogeneous agricultural areas & Agro-forestry areas & \\
\hline Forest and semi natural areas & Scrub and/or herbaceous veg. associations & Natural grassland & \\
\hline Forest and semi natural areas & Scrub and/or herbaceous veg. associations & Moors and heathland & NFR \\
\hline Forest and semi natural areas & Scrub and/or herbaceous veg. associations & Sclerophyllous vegetation & \\
\hline Forest and semi natural areas & Scrub and/or herbaceous veg. associations & Transitional woodland-shrub & \\
\hline Forest and semi natural areas & Open spaces with little or no vegetation & Beaches, dunes, sands & \\
\hline Forest and semi natural areas & Open spaces with little or no vegetation & Bare rocks & \\
\hline Forest and semi natural areas & Open spaces with little or no vegetation & Sparsely vegetated areas & \\
\hline Forest and semi natural areas & Open spaces with little or no vegetation & Burnt areas & \\
\hline Forest and semi natural areas & Open spaces with little or no vegetation & Glaciers and perpetual snow & \\
\hline Wetlands & Inland wetlands & Inland marshes & \\
\hline Wetlands & Inland wetlands & Inland marshes & \\
\hline Wetlands & Inland wetlands & Peat bogs & \\
\hline Wetlands & Maritime wetlands & Salt marshes & \\
\hline Wetlands & Maritime wetlands & Salines & \\
\hline Wetlands & Maritime wetlands & Intertidal flats & \\
\hline Water bodies & Inland waters & Water courses & \\
\hline Water bodies & Inland waters & Water bodies & \\
\hline Water bodies & Marine waters & Coastal lagoons & \\
\hline Water bodies & Marine waters & Estuaries & \\
\hline Water bodies & Marine waters & Sea and ocean & INV \\
\hline NODATA & NODATA & NODATA & \\
\hline UNCLASSIFIED & UNCLASSIFIED LAND SURFACE & UNCLASSIFIED LAND SURFACE & \\
\hline UNCLASSIFIED & UNCLASSIFIED WATER BODIES & UNCLASSIFIED WATER BODIES & \\
\hline UNCLASSIFIED & UNCLASSIFIED & UNCLASSIFIED & \\
\hline
\end{tabular}




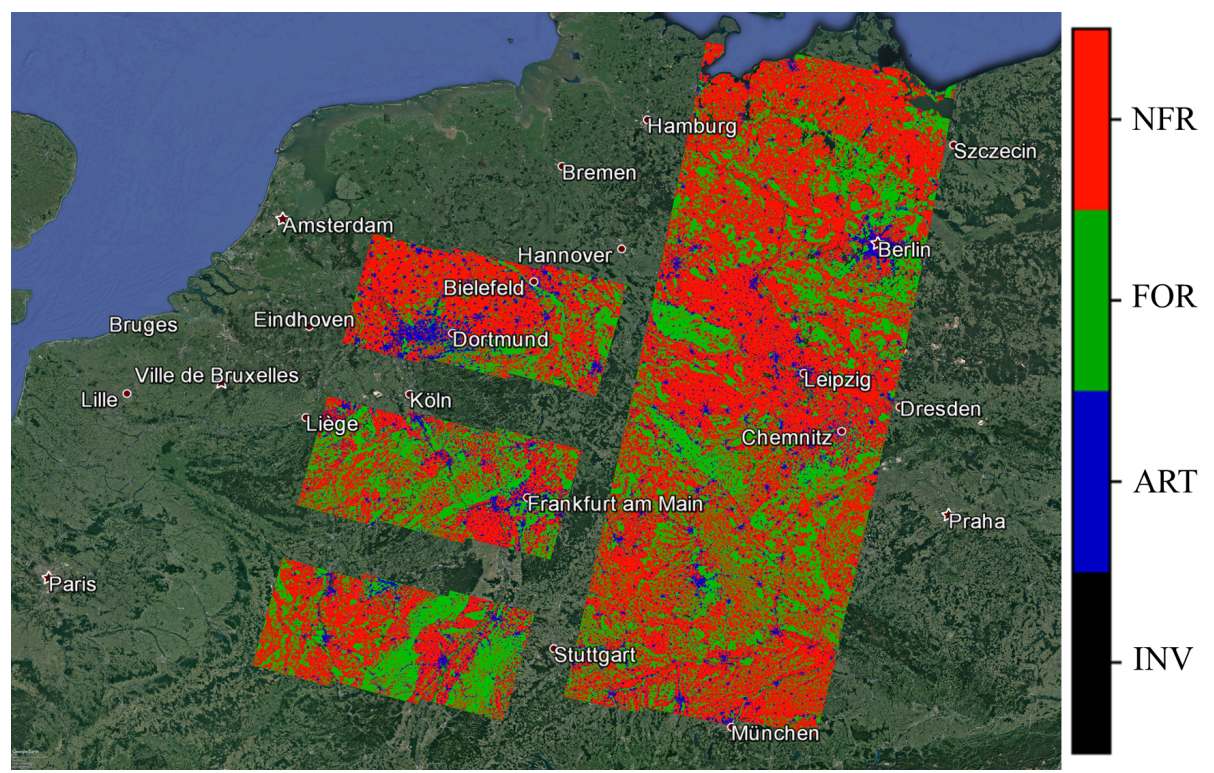

Figure 4: Reference CORINE land cover map from 2012 for the considered test sites over Europe, superimposed to an optical image from Google Earth. The original land cover classes are grouped into the higher-level ones described in table 2 (ART: artificial surfaces (blue), FOR: forests (green), NFR: non-forested areas (red), INV: water bodies and invalid or no data (black)).

higher-level classes, as shown in the last column of table 2 artificial surfaces (ART), forests (FOR), non-forested areas (NFR), and water bodies and invalid or no data (INV). We used the first three higher-level classes for performing the classification, while the last one, which includes both, water and invalid pixels, was masked out.

The decision to mask out water bodies using the CORINE associated layer resides in the fact that an analysis of temporal decorrelation evolution over such areas would not be of interest, since, typically, water completely decorrelates in 3 a very short time (much lower than 6 days). There are therefore more reliable approaches than the proposed one for mapping water surfaces, such as e.g. thresholding on backscatter low levels [44, [45], which are anyway out of the scope of the present work. 


\section{Results and discussion}

In this section, we describe the application of the developed algorithm to the Sentinel-1 data set for the parameters estimation of the temporal correlation model and we comment on the results. We present the obtained classification map and we derive its performance with respect to the external reference.

\subsection{Experimental results}

Out of the available 7 stacks, we exploited 6 of them (stack 1 to stack 6 ) for data analysis and training of the classification algorithm, and the remaining one (stack 7) for testing and performance evaluation.

\subsubsection{Estimated model parameters}

We now present the analysis and the classification results obtained by applying the algorithm presented in section 3.2 to the Sentinel-1 stacks 1-6 in table 1 . The CORINE land cover map from 2012 was used as classification reference. For each available land cover class (artificial surfaces (ART), forests (FOR), and non-forested areas NFR), we evaluate the temporally multi-looked backscatter $\hat{\gamma}^{0}$, and we perform the exponential fitting of the temporal correlation factor $\hat{\rho}_{\text {temp }}$, retrieving the $\hat{\rho}_{\mathrm{LT}}$ and $\hat{\tau}$ parameters.

We also perform a comparison among the different models presented in section 2 (equation 4 to 7 ) in terms of mean square error (MSE) between the real measurements and the fitted model. The results of this analysis have been one of the main drivers for the selection of the model to be used in our algorithm. For this specific purpose, the temporal correlation factor $\hat{\rho}_{\text {temp }}$, and not the interferometric coherence $\hat{\rho}$, is used in both models in equation 4 and 5 setting $\rho_{0}=1$. For each model, we evaluate the MSE for a set of 3000 observations, randomly selected among the three considered land cover classes (1000 samples per class). We then evaluate the mean MSE and its standard deviation. The results are summarized in table 3 . It is clear, that the introduction of the long-term coherence $\rho_{\mathrm{LT}}$ in both models 3 and 4 (equation 5 and 7) leads to a significant decrease of the MSE and, therefore, to an overall better fitting of the 
Table 3: MSE between observations and fitted models, computed using 3000 samples (1000 samples per land cover class). Four different models are considered: model 1 as in equation 4 with $\rho_{0}=1$, model 2 as in equation 5 with $\rho_{0}=1$, model 3 as in equation 6 and model 4 as in equation 7

\begin{tabular}{ccc}
\hline model & mean MSE & MSE standard deviation \\
\hline 1 & 0.047 & 0.012 \\
2 & 0.155 & 0.044 \\
3 & 0.006 & 0.008 \\
4 & 0.005 & 0.007 \\
\hline
\end{tabular}

data. Finally, model 4 shows a slightly better performance than model 3 , and is therefore chosen as reference model for the present work.

The normalized histograms of the estimated quantities $\hat{\gamma}^{0}, \hat{\rho}_{\mathrm{LT}}$, and $\hat{\tau}$ are depicted in figure 5 (a) to (c) for each land cover class, separately. It can be observed that the distributions of $\hat{\gamma}^{0}$ and $\hat{\tau}$, for each single class, can be approximated by mono-modal Gaussian-like distributions with well separable mean values but with a significant overlapping, expecially for the $\hat{\tau}$ distribution. On the other hand, the distributions of $\hat{\rho}_{\mathrm{LT}}$ for the classes forests (FOR) and non-forested areas (NFR) are largely superimposed, while a high degree of separation is visible between artificial surfaces (ART) and all other classes.

Figure 5 (d) shows the derived models of the temporal correlation factor $\hat{\rho}_{\text {temp }}$ in equation 7, obtained by applying the mean values of the distributions of $\hat{\rho}_{\mathrm{LT}}$ and $\hat{\tau}$. As expected, the artificial surfaces (ART) class decorrelates much less with respect to the other two classes. This is due to the intrinsic nature of artificial scatterers, whose radar cross-section and phase are more stable in time with respect to distributed ones.

It has to be noted that a meaningful use of multiple features as input to a classifier requires a low degree of correlation among them. In order to verify this aspect, for each land cover class, we compute the bi-dimensional histograms of all possible parameters combinations. The results are depicted in figure 6 . From the histograms orientation, we notice that no relevant correlation between 


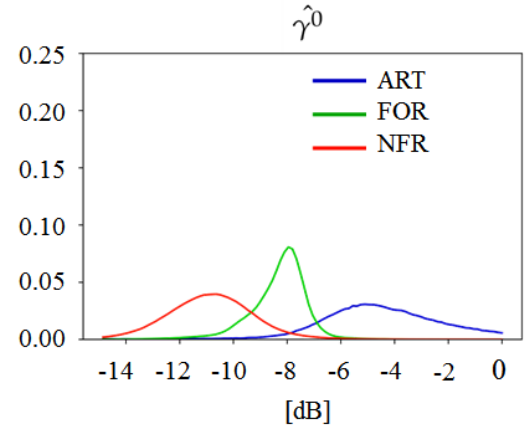

(a)

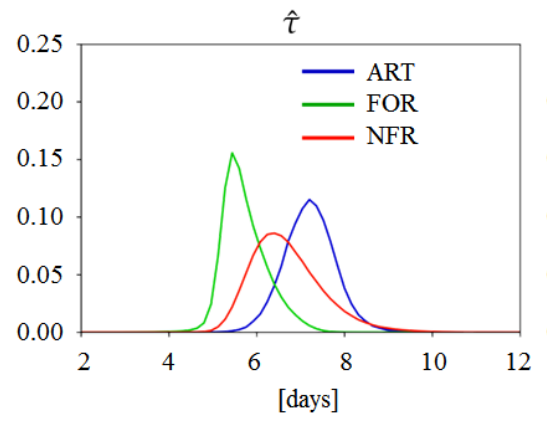

(c)

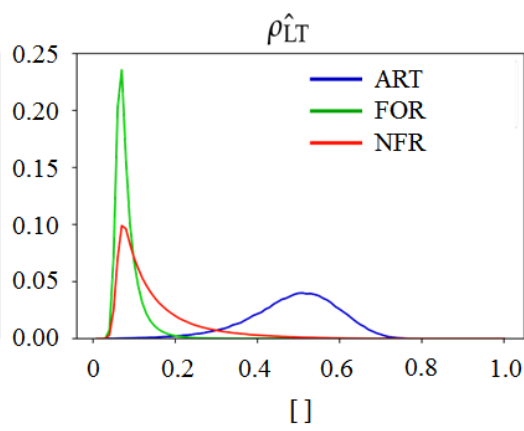

(b)

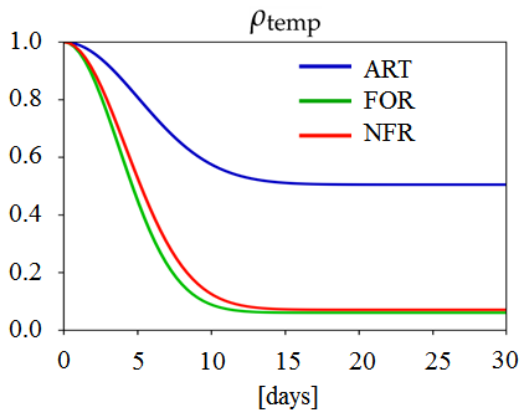

(d)

Figure 5: (a) Normalized histogram of the temporal multi-looked backscatter $\hat{\gamma}^{0}$. (b) and (c) Normalized histograms of the exponential fitting parameters $\hat{\rho}_{\mathrm{LT}}$ and $\hat{\tau}$, respectively. (d) Exponential model of the volume correlation factor, derived using the mean values of $\hat{\rho}_{\mathrm{LT}}$ and $\hat{\tau}$ distributions. Three land cover classes are considered: artificial surfaces (ART) (blue), forests (FOR) (green), and non-forested areas (NFR) (red).

features is observed.

\subsubsection{Classification results and performance analysis}

In the following we show the results, obtained by applying the algorithm described in section 3.4, and we analyze its behaviour in the proposed three different cases, characterized by different features as input to the RF classifier:

- case 1: $\hat{\gamma}^{0}$ and $\theta_{\text {inc }}$,

- case 2: $\hat{\tau}, \hat{\rho}_{\mathrm{LT}}$, and $\theta_{\mathrm{inc}}$, 

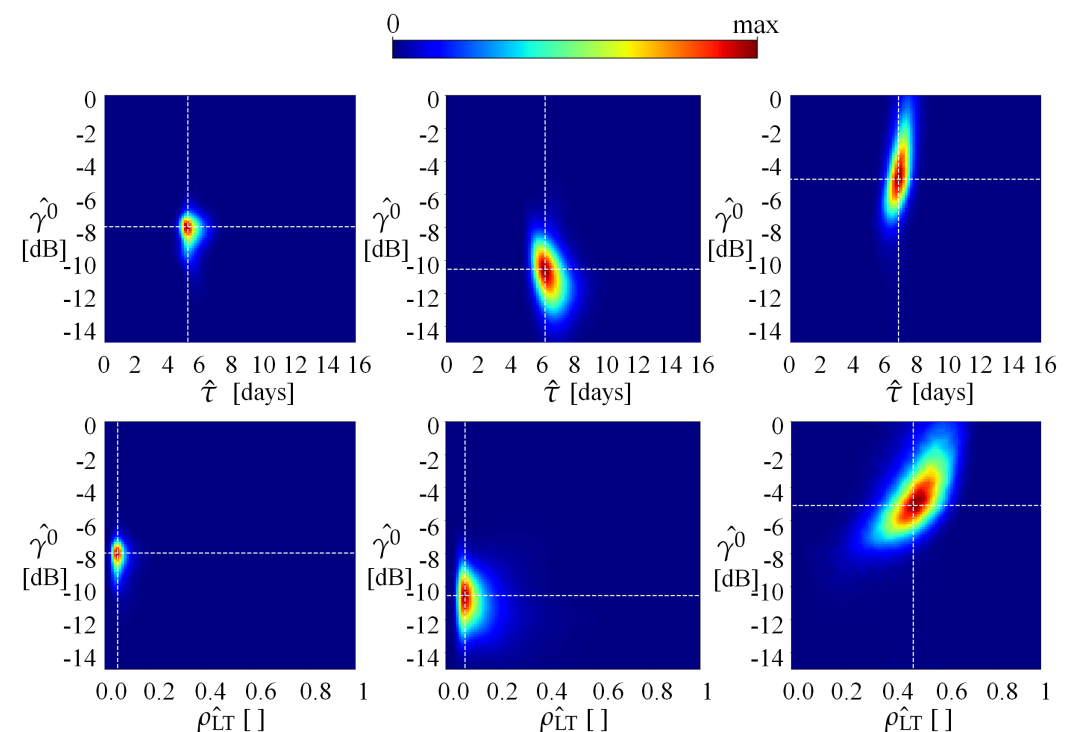

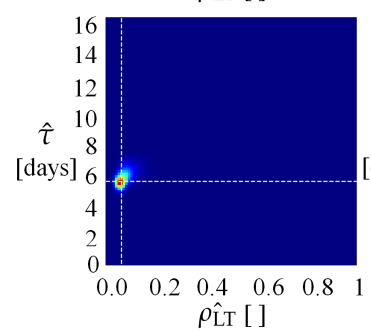

(a)

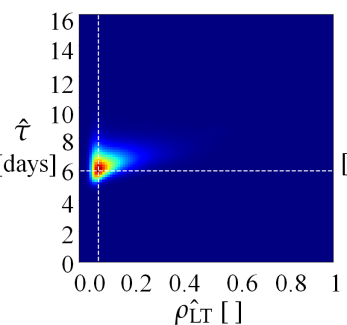

(b)

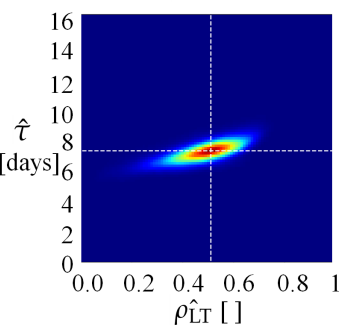

(c)

Figure 6: Normalized two-dimensional histograms of $\hat{\gamma}^{0}, \hat{\tau}$, and $\hat{\rho}_{\mathrm{LT}}$, for the land cover classes: forests (FOR) (a), non-forested areas (NFR) (b), and artificial surfaces (ART) (c).

- case 3: $\hat{\gamma}^{0}, \hat{\tau}, \hat{\rho}_{\mathrm{LT}}$, and $\theta_{\mathrm{inc}}$.

Figure 7 shows the derived classification map from stack 1 for case 3, where both backscatter and interferometric parameters are used as input features.

As already mentioned, the number of trees $n_{e s t}$ and the minimum number of sample in a leaf node lea $f_{\text {size }}$ are set to 50 . We based the choice of such values on the evaluation of the overall accuracy $A$ for the suggested input features configuration, case 3. The results, presented in figure 8, are coherent with the theory behind the RF: usually, the higher the number of trees, the better the 


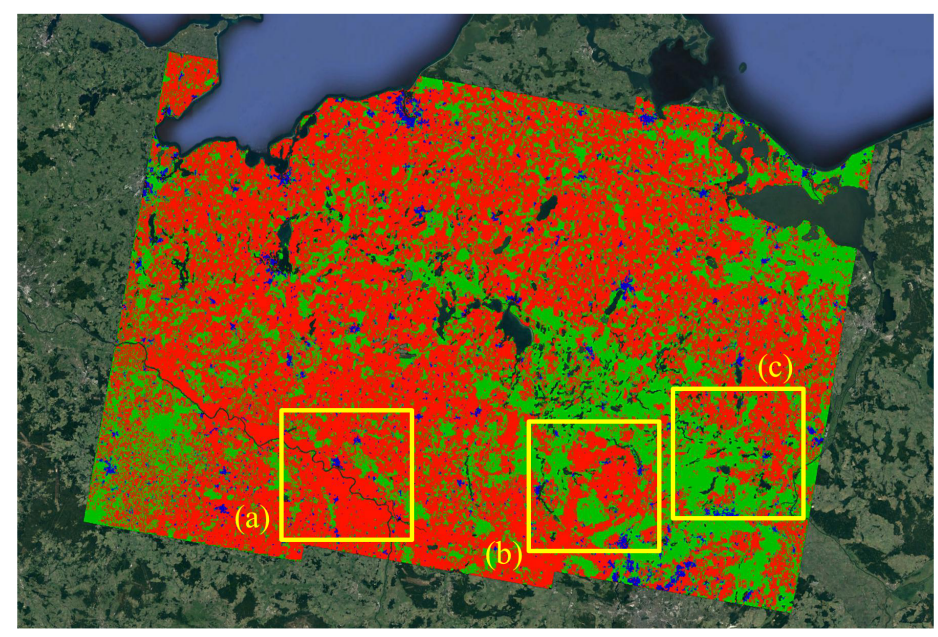

口 ART $\square$ FOR $\square$ NFR

Figure 7: Derived classification map for the test Sentinel-1 stack 7, superimposed to Google Earth. Yellow polygons identify three patches which are displayed in detail in figure 9 Blue: artificial surfaces (ART), green: forests (FOR), red: non-forested areas (NFR). For a better visualization on Google Earth, a transparent layer is associated to water bodies and invalid or no data samples.

412 algorithm can learn from the input data, at the cost of an increasing training 413 time. On the other hand, if the number of samples in a leaf node increases 414 too much, the model cannot learn enough about the data and we fall in an 415 underfitting case. In our results, we experienced a significant improvement in 416 terms of classification accuracy by increasing both $n_{\text {est }}$ and lea $f_{\text {size }}$, up to a ${ }_{417}$ saturation level where the RF performance stabilizes. The chosen values of $n_{\text {est }}$ and leaf $f_{\text {size }}$ equal to 50 are, on the one hand close to such a saturation level, and on the other hand a good compromise in terms of computational costs.

Let us now concentrate on the analysis of the three different patches high421 lighted in figure 7 (yellow), which are now depicted in details in figure 9. The 422 corresponding optical images, taken from Google Earth, and the reference 423 CORINE land cover map are depicted in rows (i) and (ii), respectively. The ${ }_{424}$ crops in rows (iii) to (v) correspond to the three different cases introduced in 425 section 3.4 which differ from each other depending on the input features to the 


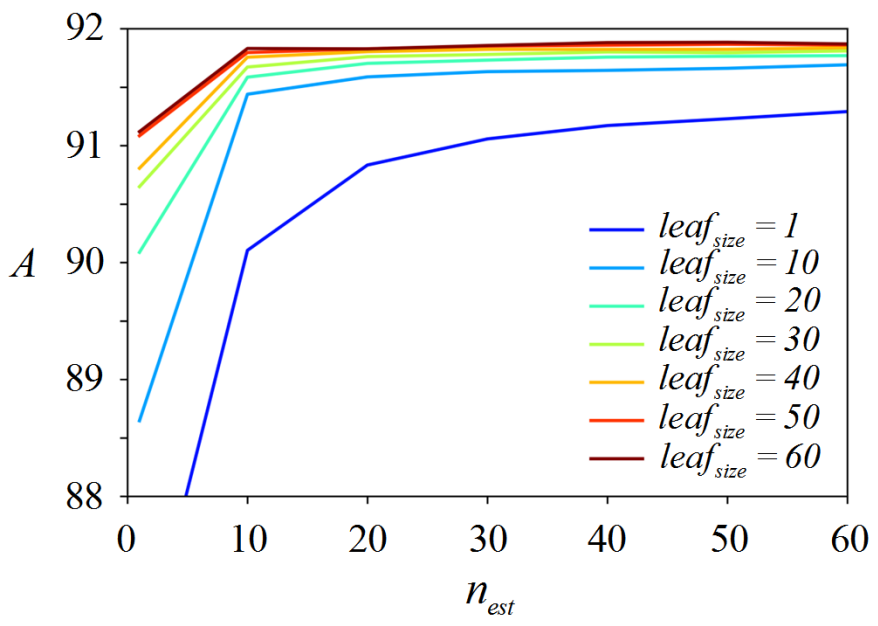

Figure 8: Overall accuracy $(A)$ as a function of two relevant RF parameters: minimum number of sample in a leaf node $\left(\right.$ lea $\left.f_{\text {size }}\right)$ and number of trees/estimators $\left(n_{\text {est }}\right)$.

RF classifier (case 1, case 2, and case 3).

From a first visual inspection, one can notice that case 1 (iii), based on the use of backscatter information only (together with the local incidence angle), tends to underestimate the artificial surfaces (ART) and the forests (FOR) classes in favour of the non-forested areas (NFR). This effect is prevalent in crop (a), but it can be observed in all the selected crops as well. On the other hand, we observe that case 2 (iv), based on the use of interferometric parameters only, shows a more reliable behaviour for all the three classes when compared to case 1. This can be clearly observed for crop (a) and (b), while the third crop shows some misclassification errors for the non-forested areas (NFR) class in favour of the forests (FOR) one. Finally, the combined use of both, backscatter and interferometric parameters (case $3(\mathrm{v})$ ), is overall less affected by the previously mentioned misclassifications, which are better solved in all crops.

Furthermore, in order to precisely assess the resulting performance, we compute the accuracy $A$ over the considered patches for all different cases. The results are summarized in table 4 and confirm the considerations derived from the visual inspection of the classified patches. 
(a)
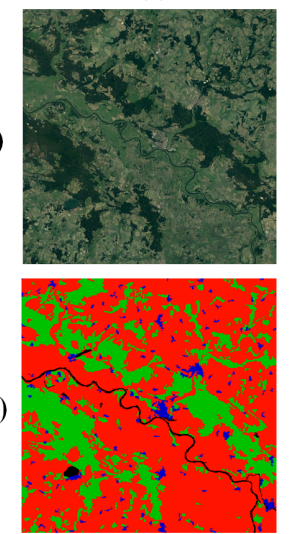

(ii)
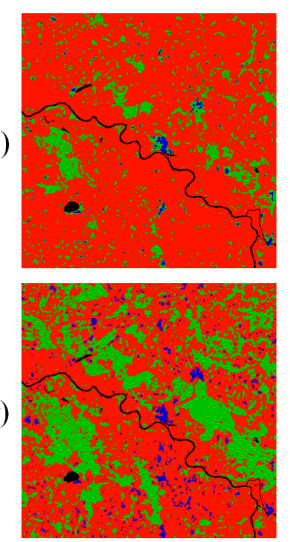

(iv)

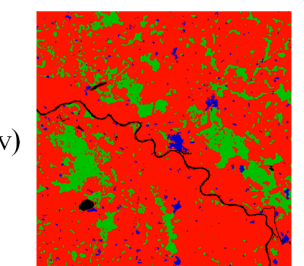

a ART FOR (b)
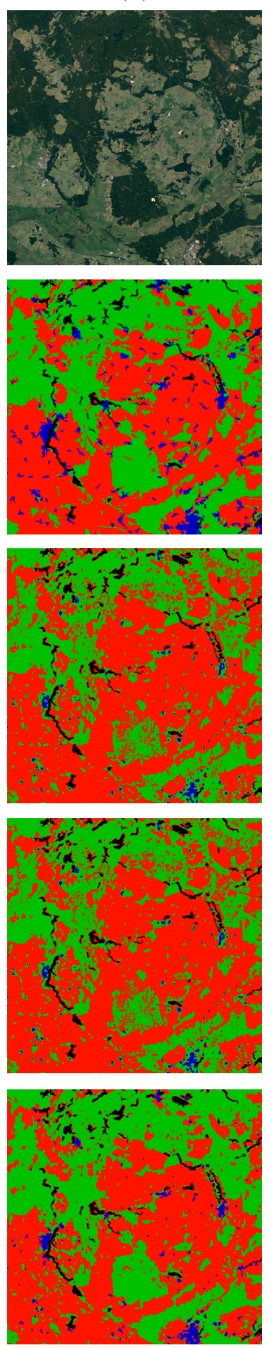

口 NFR (c)
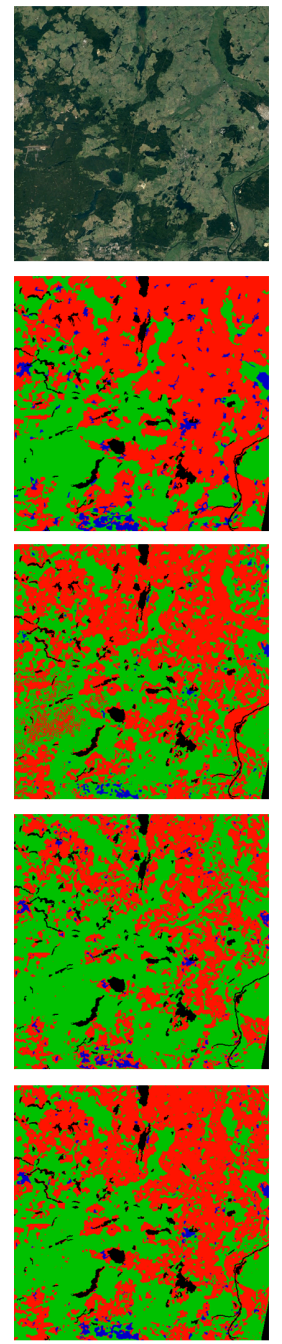

INV

Figure 9: Sample patches $(512 \times 512$ pixels) of three different locations from figure 7 (row (i)) optical image from Google Earth, (row (ii)) reference CORINE classification Map, (rows (iii), (iv), and (v)) classification maps derived from Sentinel-1 stacks for case 1 (iii), case 2 (iv), and case 3 (v) (blue: artificial surfaces (ART), green: forests (FOR), red: non-forested areas (NFR)). Water bodies and invalid pixels (INV) depicted in black and filtered out using CORINE, as described in section 3 
Table 4: Classification accuracy $A$ for the three sample patches in figure 9 characterized by heterogeneous structures: (a) Wittenberge area, (b) Neuruppin area, and (c) Angermünde area, and for 2 mln pixels randomly selected within the image (overall). Performance comparison between different input features to the RF classifier (case 1: $\left(\hat{\gamma}^{0}, \theta_{\text {inc }}\right)$, case 2: $\left(\hat{\tau}, \hat{\rho}_{\mathrm{LT}}, \theta_{\text {inc }}\right)$, case 3: $\left.\left(\hat{\gamma}^{0}, \hat{\tau}, \hat{\rho}_{\mathrm{LT}}, \theta_{\mathrm{inc}}\right)\right)$.

\begin{tabular}{ccccc}
\hline input case & patch (a) & patch (b) & patch (c) & overall \\
\hline case 1 & $76.02 \%$ & $79.93 \%$ & $76.86 \%$ & $88.73 \%$ \\
case 2 & $79.30 \%$ & $77.98 \%$ & $71.43 \%$ & $78.77 \%$ \\
case 3 & $83.28 \%$ & $86.84 \%$ & $82.9 \%$ & $91.85 \%$ \\
\hline
\end{tabular}

443 Additionally, for the three different cases, we also compute the accuracy $A$ using ${ }_{444}$ all available pixels (2.5 $\mathrm{mln}$ forests, $2.5 \mathrm{mln}$ non-forested areas, 243335 artificial surfaces (all the available ones)), randomly selected within the test image, avoiding border pixels between different classes, where the probability of classification errors within the reference map increases. The results are presented in table 4 (last column - overall). As it can be seen, the combined use of backscatter and interferometric parameters (case 3) shows the best performance, with an overall accuracy $A$ of $91.85 \%$.

\subsection{Discussion}

From the performed analysis, we observed that, when using alternatively the backscatter or the interferometric parameters, an overall comparable performance can be achieved. On the other hand, the second option (interferometric parameters only) shows a considerably better performance in presence of forests and artificial surfaces.

The reader should also be aware of the fact that the computed levels of accuracy can be subjected to classification errors within the external reference itself, as well as actual changes in the land cover, which occurred between the CORINE land cover map generation (2012) and the Sentinel-1 stacks (acquired in 2018) used for the presented analysis. 
Nevertheless, the use of the interferometric parameters $\hat{\tau}$ and $\hat{\rho}_{\mathrm{LT}}$ represents a valuable additional information with respect to the multi-temporal backscatter $\hat{\gamma}^{0}$. This can be inferred from both, the analysis of the histograms for each single land cover class (figure 5) and the results of the classification itself shown in table 4. Indeed, the classification performance is always higher when combining all input features (case 3), as proposed in our approach.

In our opinion, the use of interferometric parameters represents therefore a key aspect towards the development of a reliable land cover classification from multi-temporal interferometric SAR data, which takes into account a larger number of classes.

\section{Conclusions and outlook}

In this paper we presented a novel approach to generate large-scale land cover maps from multi-temporal InSAR short-time-series, by combining the information from both, backscatter and interferometry. The evolution in time of the temporal decorrelation can be modeled as an exponential decay, whose fitting parameters serve as input features for a machine learning classifier (in our case, the Random Forest).

The proposed methodology has been developed and tested on the example of Sentinel-1 C-band SAR data over Europe, for three land cover classes: artificial surfaces, forests, and non-forested areas. The results show an overall classification accuracy above $91 \%$.

Given the use of short-time-series, the target scene is observed for a brief interval (about one month in our analysis), and the derived maps not only can be related to a specific time frame, but they can also be generated at regular intervals: yearly, for repeating the analysis at the same seasonal conditions, or several times within a year.

The analysis of short-time-series sequences, combined in a daisy chain fashion, is a capability of paramount importance if we want to apply the method e.g. for catching nearly-real time deforestation or abrupt land cover changes. This 
is therefore a crucial asset of the approach that would be lost if we considered temporal parameters only, computed over very large time spans.

If, on the one hand, the obtained results clearly demonstrate that repeat-pass interferometry adds valuable information for the classification of basic land cover classes, on the other hand, it is also clear that this work represents the first step towards the development of an effective classification framework, which takes into account a higher number of classes.

To this purpose, we plan to further extend the proposed methodology by investigating the synergistic use of short-time-series and additional methods which consider longer time spans. This will include the analysis of coherence and backscatter variability for different polarizations and over longer time frames, in order to better capture the characteristic trends of those classes showing a seasonal-dependent behavior, such as agricultural areas.

Additionally, new strategies for the preservation of data resolution will be implemented as well, following the example in [46]. By improving the output map resolution, a larger number of samples will be available for specifically training the classifier and will support the discrimination of a higher number of classes.

Finally, we will further extend the investigated area in order to provide a proof of concept about the possibility of global coverage, by increasing the number of data and testing the limitations for large scale mapping and monitoring.

\section{Acknowledgments}

The authors would like to thank the editor and the anonymous reviewers for their highly valuable comments to improve the quality of the manuscript. The performed work has been partially financed through the HI-FIVE project, granted by the ESA Living Planet Fellowship 2018.

[1] S. Bontemps, P. Defourny, E. Van Bogaert, O. Arino, V. Kalogirou, and J. R. Perez, "GLOBCOVER 2009 Product description and validation report," tech. rep., European Space Agency, Feb. 2011. 
[2] M. Friedl, D. Sulla-Menashe, B. Tan, A. Schneider, N. Ramankutty, A. Sibley, and X. Huang, "MODIS Collection 5 global land cover: Algorithm refinements and characterization of new datasets," Remote Sensing of Environment, vol. 114, pp. 168-182, 2010.

[3] M. C. Hansen, P. V. Potapov, R. Moore, M. Hancher, S. A. Turubanova, A. Tyukavina, D. Thau, S. V. Stehamn, S. J. Goetz, T. R. Loveland, and J. R. G. Kommareddy, "High-resolution global maps of 21st century forest coverage change," Science, vol. 342, pp. 850-853, Nov. 2013.

[4] R. O. Dubayah and J. B. Drake, "Lidar remote sensing for forestry," Journal of Forestry, vol. 98, pp. 44-46, Jun. 2000.

[5] B. Mora, N. E. Tsendbazar, M. Herold, and O. Arino, Land Use and Land Cover Mapping in Europe: Practices Trends, Chapter 2, Remote Sensing and Digital Image Processing. Springer Science+Business Media, 2014.

[6] M. C. Dobson, F. T. Ulaby, and L. E. Pierce, "Land-cover classification and estimation of terrain attributes using synthetic aperture radar," Remote Sensing of Environment, vol. 51, pp. 199-214, Jan. 1995.

[7] Z. Malenovsky, H. Rott, J. Cihlar, M. E. Schaepman, G. Garcia-Santos, R. Fernandes, and M. Berger, "Sentinels for science: Potential of Sentinel-1,2, and-3 missions for scientific observations of ocean, cryosphere, and land," Remote Sensing of Environment, vol. 120, pp. 91-101, 2012.

[8] M. Ruetschi, M. E. Schaepman, and D. Small, "Using multi-temporal Sentinel-2 C-band backscatter to monitor phenology and classify deciduous and coniferous forests in northern Switzerland," Remote Sensing, vol. 10, pp. 1-30, Jan. 2018.

[9] M. Shimada, T. Itoh, T. Motooka, M. Watanabe, T. Shiraishi, R. Thapa, and R. Lucas, "New global forest/non-forest maps from ALOS PALSAR data (2007-2010)," Remote Sensing of Environment, vol. 155, pp. 13-31, 2014. 
[10] U. Wertmuller and C. L. Werner, "SAR interferometric signatures of forest," IEEE Transactions on Geoscience and Remote Sensing, vol. 33, pp. 11531161, Sep. 1995.

[11] T. Strozzi, P. Dammert, U. Wegmüller, J.-M. Martinez, J. I. H. Askne, A. Beaudoin, and N. T. Hallikainen, "Landuse mapping with ERS SAR interferometry," IEEE Transactions on Geoscience and Remote Sensing, vol. 38, pp. 766-775, Mar. 2000.

[12] L. Bruzzone, M. Marconcini, U. Wertmuller, and A. Wiesmann, "An Advanced System for the Automatic Classification of Multitemporal SAR images," IEEE Transactions on Geoscience and Remote Sensing, vol. 42, pp. 1321-1334, Jun. 2004.

[13] A. A. Okhimamhe, "ERS SAR interferometry for land cover mapping in a savanna area in Africa," International Journal of Remote Sensing, vol. 120, pp. 3583-3594, May 2010.

[14] D. Geudtner, R. Torres, P. Snoeij, A. Ostergaard, I. Navas-Traver, B. Rommen, and M. Brown, "Sentinel-1 system overview and performance," Living Planet Symposium, 2013.

[15] K. Chureesampant and J. Susaki, "Land cover classification using multitemporal SAR data and optical data fusion with adaptive training sample selection," in 2012 IEEE International Geoscience and Remote Sensing Symposium, pp. 6177-6180, July 2012.

[16] H. Zebker and J. Villasenor, "Decorrelation in interferometric radar echoes," IEEE Transactions on Geoscience and Remote Sensing, vol. 30, no. 5, pp. 950-959, 1992.

[17] G. Krieger, A. Moreira, H. Fiedler, I. Hajnsek, M. Werner, M. Younis, and M. Zink, "TanDEM-X: A satellite formation for high-resolution SAR interferometry," IEEE Transactions on Geoscience and Remote Sensing, vol. 45, no. 11, 2007. 
[18] M. Martone, P. Rizzoli, C. Wecklich, C. Gonzalez, J.-L. Bueso-Bello, P. Valdo, D. Schulze, M. Zink, G. Krieger, and A. Moreira, "The Global Forest/Non-Forest Map from TanDEM-X Interferometric SAR Data," Remote Sensing of Environment, vol. 205, pp. 352-373, Feb. 2018.

[19] M. Martone, F. Sica, C. Gonzalez, J.-L. Bueso-Bello, P. Valdo, and P. Rizzoli, "High-Resolution Forest Mapping from TanDEM-X Interferometric Data Exploiting Nonlocal Filtering," Remote Sensing, vol. 10, p. 1477, Sep. 2018.

[20] P. Rizzoli, M. Martone, H. Rott, and A. Moreira, "Characterization of Snow Facies on the Greenland Ice Sheet Observed by TanDEM-X Interferometric SAR Data," Remote Sensing, vol. 9, Mar. 2017.

[21] F. Rocca, "Modeling interferogram stacks," IEEE Transactions on Geoscience and Remote Sensing, vol. 45, no. 10, pp. 3289-3299, 2007.

[22] A. Parizzi, X. Y. Cong, and M. Eineder, "First Results from Multifrequency Interferometry. a Comparison of Different Decorrelation Time Constants at L, C and X Band," ESA Special Publication, vol. 677, 2010.

[23] R. M. Goldstein and H. A. Zebker, "Interferometric radar measurement of ocean surface currents," Nature, vol. 328, no. 6132, pp. 707-709, 1987.

[24] A. Moccia and G. Rufino, "Spaceborne along-track SAR interferometry: Performance analysis and mission scenarios," IEEE Transactions on Aerospace and Electronic systems, vol. 37, no. 1, pp. 199-213, 2001.

[25] N. Yague-Martinez, P. Prats-Iraola, F. R. Gonzalez, R. Brcic, R. Shau, D. Geudtner, M. Eineder, and R. Bamler, "Interferometric processing of Sentinel-1 TOPS data," IEEE Transactions on Geoscience and Remote Sensing, vol. 54, no. 4, pp. 2220-2234, 2016.

[26] M. Nannini, P. Prats-Iraola, R. Scheiber, N. Yague-Martinez, F. Minati, F. Vecchioli, M. Costantini, S. Borgstrom, P. De Martino, V. Siniscalchi, T. Walter, M. Foumelis, and Y. L. Desnos, "Sentinel-1 mission: results of the 
InSARap project," EUSAR 2016: 11th European Conference on Synthetic Aperture Radar, 2016.

[27] D. Small, "Flattening gamma: Radiometric terrain correction for SAR imagery," IEEE Transactions on Geoscience and Remote Sensing, vol. 49, no. 8, 2011.

[28] R. K. Raney, A. Freeman, R. W. Hawkins, and R. Bamler, "A plea for radar brightness," International Geoscience and Remote Sensing Symposium, vol. 2, pp. 1090-1090, 1994.

[29] M. Bourbigot, H. Johnsen, and R. Piantanida, "Sentinel Product Definition," Tech. Rep. S1-RS-MDA-52-7440, European Space Agency (ESA), March 2016.

[30] F. Gatelli, A. M. Guamieri, F. Parizzi, P. Pasquali, C. Prati, and F. Rocca, "The wavenumber shift in SAR interferometry," IEEE Transactions on Geoscience and Remote Sensing, vol. 32, no. 4, 1994.

[31] R. Touzi, A. Lopes, J. Bruniquel, and P. W. Vachon, "Coherence estimation for SAR imagery," IEEE Transactions on Geoscience and Remote Sensing, vol. 37 , no. 1, pp. 135-149, 1999.

[32] R. Piantanida, N. Miranda, N. Franceschi, and P. Meadows, "Thermal Denoising of Products Generated by the S-1 IPF," Tech. Rep. MPC-0392, European Space Agency (ESA), Nov. 2017.

[33] E. Attema, C. Cafforio, M. Gottwald, P. Guccione, A. M. Guarnieri, F. Rocca, and P. Snoeij, "Flexible dynamic block adaptive quantization for Sentinel-1 SAR missions," IEEE Geoscience and Remote Sensing Letters, vol. 7, no. 4, pp. 766-770, 2010.

[34] D. Giudici, "GMES Sentinel-1 SAR Performance Analysis," Tech. Rep. S1.TN-ARE-PL-0001 version 1.7, European Space Agency, Mar. 2013. 
[35] M. Martone, P. Rizzoli, and G. Krieger, "Volume decorrelation effects in TanDEM-X interferometric SAR data," IEEE Geoscience and Remote Sensing Letters, vol. 13, no. 12, pp. 1812-1816, 2016.

[36] L. Breiman, "Random forests," Machine Learning, vol. 45, pp. 5-32, Oct. 2001.

[37] M. Belgiu and L. Dragut, "Random forest in remote sensing: A review of applications and future directions," ISPRS Journal of Photogrammetry and Remote Sensing, vol. 114, pp. 24-31, 2016.

[38] V. F. Rodriguez-Galiano, B. Ghimire, J. Rogan, M. Chica-Olmo, and J. P. Rigol-Sanchez, "An assessment of the effectiveness of a random forest classifier for land-cover classification," ISPRS Journal of Photogrammetry and Remote Sensing, vol. 67, pp. 93-104, 2012.

[39] N. Horning, "Random Forests: An algorithm for image classification and generation of continuous fields data sets," In Proceedings of the International Conference on Geoinformatics for Spatial Infrastructure Development in Earth and Allied Sciences, vol. 911, Dec. 2010.

[40] A. D. Kulkarni and B. Lowe, "Random forest algorithm for land cover classification," International Journal on Recent and Innovation Trends in Computing and Communication, vol. 4, no. 3, pp. 58-63, 2016.

[41] B. Waske and M. Braun, "Classifier ensembles for land cover mapping using multitemporal SAR imagery," ISPRS Journal of Photogrammetry and Remote Sensing, vol. 64, no. 5, pp. 450-457, 2009.

[42] L. Breiman, J. H. Friedman, R. A. Olshen, and C. J. Stone, Classification and regression trees. Chapman and Hall, 1984.

[43] C. S. G. Jaffrain, "CORINE Land Cover 2012 Final Validation Report," Tech. Rep. GIO_CLC2012_Validation_Report_SC2_1_4, European Environment Agency, Feb 2017. 
[44] M. Santoro and U. Wegmüller, "Multi-temporal Synthetic Aperture Radar Metrics Applied to Map Open Water Bodies," IEEE Journal of Selected Topics in Applied Earth Observations and Remote Sensing, vol. 7, pp. 32253238, Aug 2014.

[45] L. Landuyt, A. Van Wesemael, G. J.-P. Schumann, R. Hostache, N. E. C. Verhoest, and F. M. B. van Coillie, "Flood Mapping Based on Synthetic Aperture Radar: An Assessment of Established Approaches," IEEE Tansaction on Geoscience and Remote Sensing, vol. 57, pp. 722-739, Feb 2019.

[46] F. Sica, D. Reale, G. Poggi, L. Verdoliva, and G. Fornaro, "Nonlocal adaptive multilooking in SAR multipass differential interferometry," IEEE Journal of Selected Topics in Applied Earth Observations and Remote Sensing, vol. 8, no. 4, pp. 1727-1742, 2015. 